\title{
The BU72- $\mu$ opioid receptor crystal structure is a covalent adduct
}

\author{
Thomas A. Munro \\ School of Life and Environmental Sciences, Deakin University, Melbourne, Australia \\ t.munro@deakin.edu.au \\ orcid.org/0000-0002-3366-7149
}

In the crystal structure of BU72 bound to the $\mu$ opioid receptor $(\mu \mathrm{OR})$, the

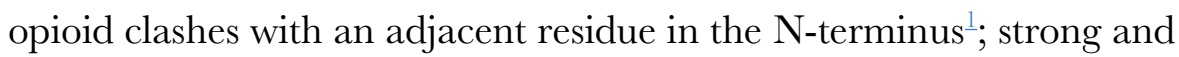
unexplained electron density connects the two, centered on a point $\sim 1.6 \AA$ from each. This is too short for non-covalent interactions, implying covalent bonds to an unmodeled non-hydrogen atom. A magnesium ion has recently been proposed as a candidate ${ }^{2}$. However, this would require unrealistically short bonds and an incomplete coordination shell. Moreover, the crystals were prepared without magnesium salts, but with components that can generate reactive oxygen species (ROS): HEPES buffer, nickel ions, and an N-terminus that forms redox-active nickel complexes. Here I show that an oxygen atom fits the unexplained density well, giving a type of covalent adduct known to form in the presence of ROS, with reasonable geometry and no clashes. While the precise structure is tentative, the observed density firmly establishes covalent bonds linking ligand and residue. Severe strain is evident in the ligand, the tethered N-terminus, and the connecting bonds. This strain, along with interactions between the N-terminus and surrounding residues, is likely to distort the receptor conformation. The subsequent $\mu \mathrm{OR}-\mathrm{G}_{\mathrm{i}}$ structure $^{3}$, which differs in several features associated with activation, is therefore likely to be a more accurate model of the active receptor. The possibility of reactions like this should be considered in the choice of protein truncation sites and purification conditions. 

unexpectedly intrudes into the binding pocket ${ }^{1}$. The third residue of the terminus, His54, clashes with the secondary amine of BU72, with an $\mathrm{N} \cdots \mathrm{N}$ overlap of $0.56 \AA$ (Figure 1a). The region of overlap is also filled with unexplained electron density (up to $6.8 \sigma$ ), centered on a point very close to both $\mathrm{N}$ atoms $(\sim 1.6 \AA)$. This is much too close for non-covalent interactions $(\geq 2.4$ $\AA)^{4}$, which would also not give uninterrupted electron density between the $\mathrm{N}$ atoms (Figure 1d). Thus, both atoms must be covalently bonded to an unmodeled non-hydrogen atom. The recent revision of the stereochemistry of BU72 did not resolve these problems ${ }^{5,6}$.

Figure 1. Comparative fit of models to electron density.
a) original (PDB 5C1M)
b) $\mathrm{Mg}^{2+}$ complex
c) adduct

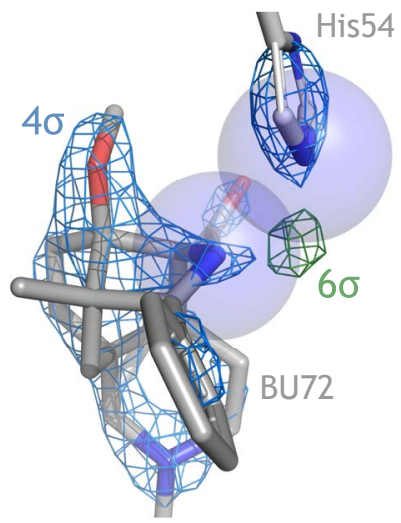

d) original $(5 \mathrm{C} 1 \mathrm{M})$

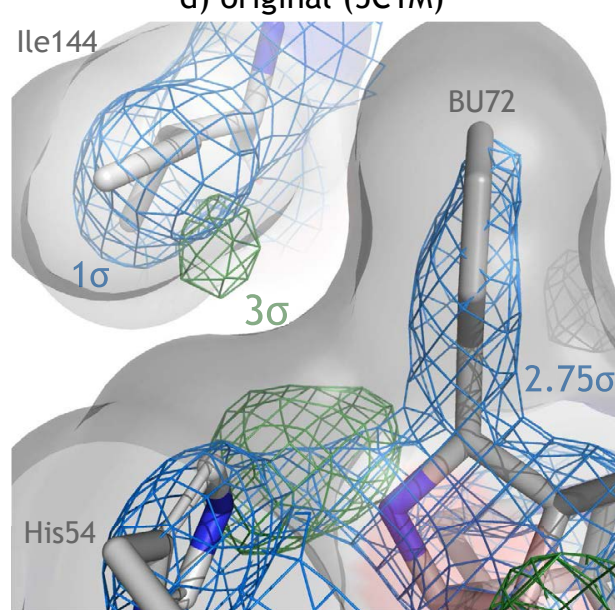

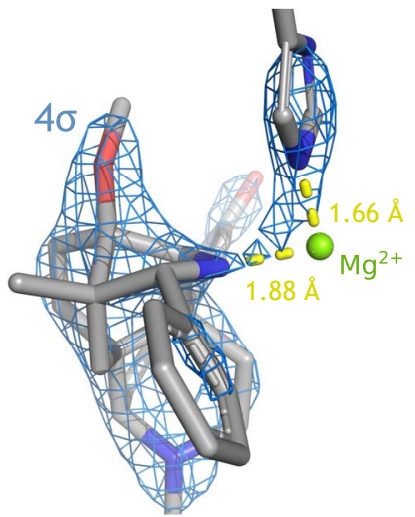

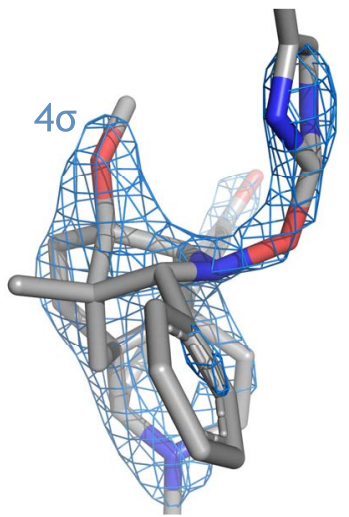

e) adduct

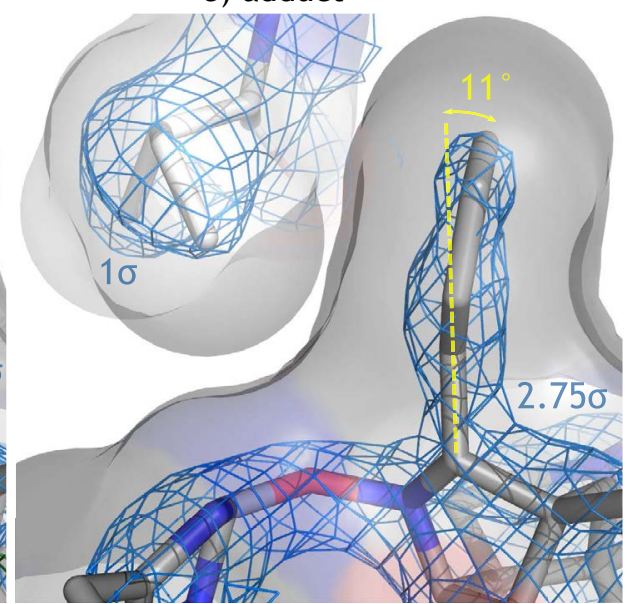

$2 F_{\mathrm{O}}-F_{\mathrm{C}}$ isomesh (blue) and $F_{\mathrm{O}}-F_{\mathrm{C}}$ omit isomesh (unexplained density in green) are shown at the indicated levels. Clashing $\mathrm{N}$ atoms are shown as spheres in (a); solventaccessible surfaces are shown in (d) and (e). 
A coordinated magnesium ion has recently been proposed as a candidate for the missing atom ${ }^{2}$. This particular metal was reportedly optimal; lithium failed to fill the density, while sodium, nickel, and zinc gave an excess. I confirmed that $\mathrm{Mg}^{2+}$ gave an excellent fit, with no excess or unexplained density above $2.5 \sigma$ (Figure 1b; see Supplementary Information for methods). However, the required $\mathrm{N} \cdots \mathrm{Mg}$ distances were unrealistic (1.88 and 1.66 $\AA$ ). Compared with values from structures of subatomic resolution $(2.19 \pm 0.06 \AA \text {, mean } \pm \sigma)^{7}$, these distances are extreme outliers, with $\mathrm{Z}$ scores of -5.2 and -8.8, respectively. Note also that $\mathrm{Mg}^{2+}$ is not centered in the density even with these unrealistically short distances, suggesting that the actual bonds must be even shorter (Figure 1b). Furthermore, the ion's coordination shell is incomplete, with a coordination number of two rather than the expected four to $\operatorname{six}^{8}$. Finally, no source of magnesium was used in the purification and crystallization of the ligandreceptor complex ${ }^{1}$. Collectively, this evidence makes this proposal untenable.

The only metal present in the buffers, sodium, gave a worse fit to the density $^{2}$, and can also be excluded due to even longer $\mathrm{N} \cdots \mathrm{Na}$ distances $(2.46 \pm$ $0.02 \AA)^{\underline{7}}$. Nickel was used for affinity chromatography, and $\mathrm{N} \cdots \mathrm{Ni}$ distances can be shorter $(1.88 \pm 0.03 \AA$ ) . However, as noted above, nickel fitted very poorly, with substantial excess electron density ${ }^{2}$; further evidence against nickel and other heavy metals is the lack of anomalous scattering noted in the original report $^{1}$. Indeed, no metal forms coordination bonds to $\mathrm{N}$ shorter than $1.76 \AA^{\underline{7}}$.

If not a metal, the missing atom must be a non-metal approximately isoelectronic with $\mathrm{Mg}^{2+}$, such as oxygen. Consistent with this possibility, the experimental conditions used can generate reactive oxygen species. The buffers used for receptor purification and crystallization contained HEPES, which generates hydrogen peroxide on exposure to light ${ }^{9}$. Additionally, the truncated N-terminus Gly-Ser-His, like other Gly-X-His N-termini, forms nickel coordination complexes $^{10}$. Specifically, Gly-Ser-His can capture nickel ions from affinity columns (e.g. PDB $\underline{1 J V N})^{11}$, which were used for purification of the receptor in this case. The resulting complexes catalyze the decomposition of hydrogen peroxide to $\operatorname{ROS}^{10}$. This may lead to unexpected reactions; ROS can 
oxidize secondary amines ${ }^{12}$ and histidine ${ }^{13}$, which contact the unexplained density in this case. Finally, the resulting radicals can be quenched by bond formation $\frac{13}{}$.

A related adduct reported recently $\frac{13}{13}$ (Figure $2 \mathrm{a}$ ) suggested a potential structure for an oxygen-bridged adduct (Figure 2b). Potential intermediates (Noxyl and histidyl radicals) are also shown, but are necessarily speculative. The proposed adduct was fitted to the binding site and refined (see Supplementary Information for methods). The adduct gave an excellent fit, with no excess or unexplained density even at $2 \sigma$ (Figure 1c). Both bonds to the oxygen atom were resolved at $4.2 \sigma$ - that is, higher density than most of the ligand itself and surrounding side-chains. The oxygen atom was well centered in the density, unlike $\mathrm{Mg}^{2+}$.

Figure 2. Proposed adduct structure and intermediates.

a) previously-reported adduct

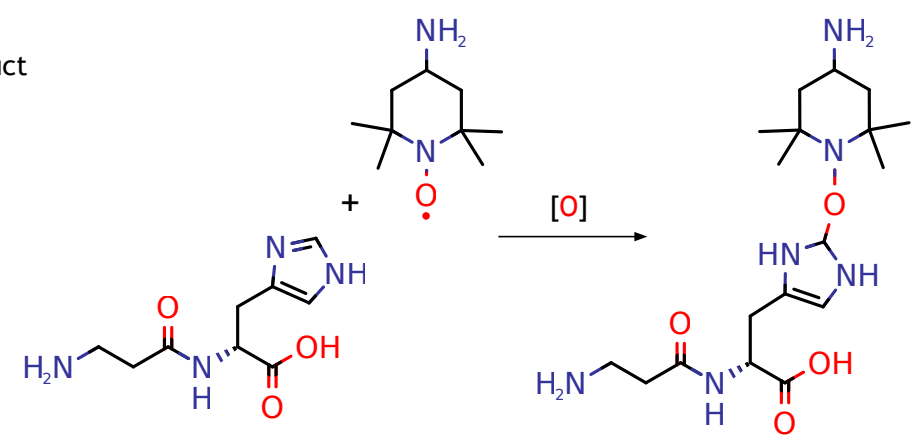

b) adduct proposed here, with possible intermediates

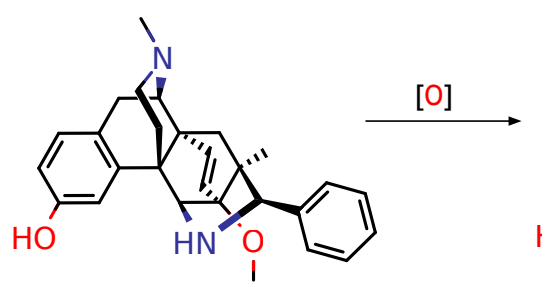

BU72 (revised structure)

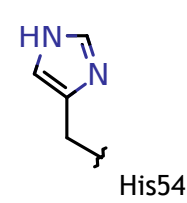

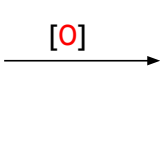
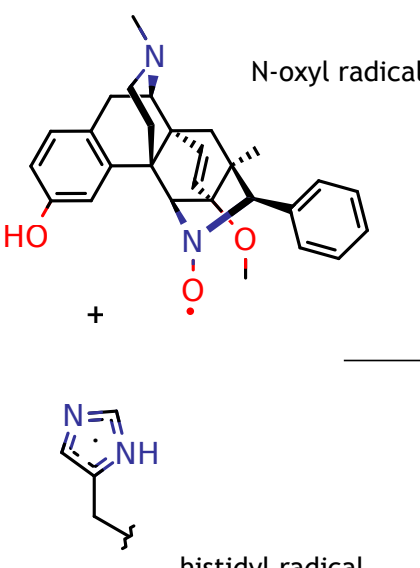

histidyl radical

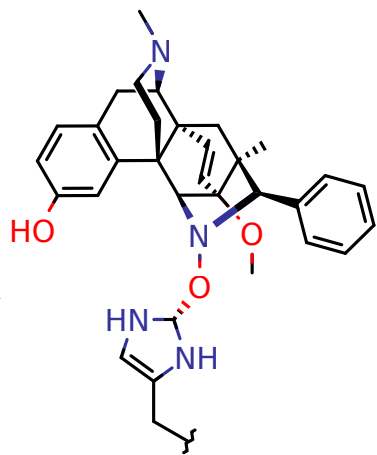

BU72-histidine adduct

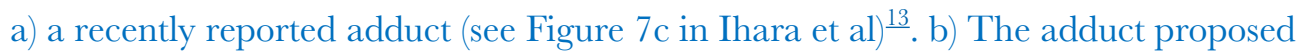
here, with possible intermediates. 
The geometry of the adduct exhibited more outliers than the revised structure of BU72 fitted in isolation, but fewer than the original structure, and gave acceptable metrics (Table 1). The only severe outlier was the bond angle at the bridging oxygen atom $\left(131^{\circ}\right.$ vs the ideal $\left.109^{\circ}: Z=7.2\right)$. There are several indications that this is real strain rather than a fitting artefact, however. The angle is clearly resolved at high density, and is consistent with tension from the tethered N-terminus. The same tension is implied by the phenyl group, which is bent out-of-plane, as if being pulled against Ile144 (Figure 1e); this bend is clearly resolved, and gives a more complementary fit to that residue than the original model (Figure 1d). Furthermore, strain is evident in the N-terminus itself: in both this model and the original, Thr60 adopts a rare and high-energy cis-peptide bond, surrounded by many clashes along the peptide backbone (Figure S2).

Table 1. Geometry relative to GRADE restraints, and fit to electron density from PDB validation.

\begin{tabular}{l|rrr} 
& original & revised & \\
\hline Structure & BU72 & BU72 & Adduct \\
Non-hydrogen atoms & 32 & 32 & 44 \\
Geometric outliers $(|\mathrm{Z}|>2)$ & 26 & 0 & 10 \\
Severe outliers $(|\mathrm{Z}|>$ 5) & 9 & 0 & 1 \\
Bond angle root mean square Z (RMSZ) & 3.23 & 0.66 & 1.52 \\
Bond length root mean square Z (RMSZ) & 3.32 & 0.38 & 1.13 \\
& & & \\
Real-space correlation coefficient $(\mathrm{RSCC}) *$ & 0.914 & 0.953 & 0.951 \\
Real-space $R(\mathrm{RSR})$ & 0.090 & 0.088 & 0.081
\end{tabular}

Lower values are better except for RSCG $(*)$.

The strain on the N-terminus is transmitted to transmembrane helix 1 (TM1), while the ligand is pulled against TM3; these forces will affect the receptor conformation. Compounding this, the $\mathrm{N}$-terminus makes numerous strong contacts throughout the binding pocket, including a dense network of 
polar contacts with TM2, TM3, and extracellular loop 2 (ECL2, Figure S2).

These contacts would also be expected to influence the receptor conformation.

A further influence is the intracellular binding partner used for the BU72- $\mu \mathrm{OR}$ structure, the G-protein mimetic nanobody Nb39. Nanobodies are known to yield slightly different receptor conformations than G-proteins ${ }^{14}$.

Consistent with these expected effects, differences are apparent in the subsequent structure of active $\mu \mathrm{OR}$ bound to $\mathrm{G}_{\mathrm{i}}$ protein $^{3}$. The intracellular end of TM 6 shifts outwards during activation; this shift is $3 \AA$ larger in the $\mu \mathrm{OR}-\mathrm{G}_{\mathrm{i}}$ structure (Figure S3) ${ }^{3}$. This difference appears to be largely due to Nb39, since the subsequent structure of $\kappa \mathrm{OR}$ bound to the same nanobody ${ }^{15}$ shows the same small shift (Figure S3). As expected from the greater distance between TM5 and TM6, several conserved interactions between them that are involved in activation $^{\underline{14}}$ are markedly different in the $\mu \mathrm{OR}-\mathrm{G}_{\mathrm{i}}$ structure (Figure S4a). Here again, the BU72- $\mu \mathrm{OR}-\mathrm{Nb} 39$ structure is more similar to kOR-Nb39 (Figure $\mathrm{S} 4 \mathrm{~b})$. Although some motifs involved in activation show very similar conformations in the $\mu \mathrm{OR}-\mathrm{G}_{\mathrm{i}}$ structure (see Figure 2c in Koehl et $\left.a l\right)^{3}$, others differ considerably (notably NPxxY ${ }^{14}$, Figure S5a). Several of these residues also show substantial differences from kOR-Nb39 (Figure S5b). Whether due to the influence of the adduct, the nanobody or both, these differences from the $\mu \mathrm{OR}$ $\mathrm{G}_{\mathrm{i}}$ structure are artefacts, and the latter is likely to be a more accurate template for modelling the active receptor.

In the original study, a search for alternative ligands to account for the unexplained density was unsuccessful. The mass spectrum of the crystallization mixture revealed a molecular ion consistent with BU72, but no others of similar mass ${ }^{\underline{1}}$. However, the intact adduct would not be detectable in solution, and one decomposition product per binding site would yield negligible concentrations relative to saturating BU72. An alternative test would be for modification of His54: proteolysis of the receptor and mass spectrometry of the fragments should reveal either the adduct or a decomposition product. Adducts of this kind tend to be unstable in the presence of ROS. The adduct mentioned above was not isolated, but detected only by mass spectrometry $\frac{13}{}$. However, in the 
present case adduct formation would liberate the nickel ion, ending the catalytic cycle and preventing further reaction.

The possibility of reactions like the one described here should be considered in the choice of truncation site and purification conditions for protein isolation. Another study using similar conditions (the related buffer PIPES, nickel affinity chromatography, and a Gly-Ser-His N-terminus) unexpectedly observed partial decomposition of the ligand ${ }^{11}$. Generation of ROS and consequent reactions could be prevented in both cases by choosing a different truncation site or buffer.

The formation of an adduct provides a simple explanation for a puzzling result in the original report: despite the extremely strong interaction implied by the structure itself, removal of the side chain of His54 by receptor mutagenesis had no detectable effect on the affinity or potency of BU72 ${ }^{\perp}$. Since the fulllength receptor used in those binding assays lacks the Gly-Ser-His N-terminus, the mechanism proposed here could not occur, and thus binding would be unaffected by the presence or absence of His54.

In conclusion, the density observed between BU72 and the receptor is not consistent with non-covalent interactions or a metal coordination complex, and must instead represent covalent bonds to a non-metal atom, approximately isoelectronic with $\mathrm{Mg}^{2+}$. The density firmly establishes the presence of this atom and two covalent bonds, along with their approximate length and geometry. While this evidence does not unambiguously identify the element, oxygen fits all these criteria. The presence of conditions known to generate ROS, along with a prior report of ROS-mediated adduct formation, suggest a plausible tentative structure and intermediates. Given that the strain within the N-terminus and its interactions with surrounding residues are likely to affect the receptor conformation, the $\mu \mathrm{OR}-\mathrm{G}_{\mathrm{i}}$ structure is likely to be a more accurate model of the active receptor. The possibility of reactions like this should be considered in the choice of truncation site and purification conditions for protein isolation. 
Acknowledgment: Robbie Joosten kindly modified the code of PDBREDO server to enable refinement of the adduct.

Supplementary Information: Methods; supplementary figures; coordinates (mmCif), structure factors (MTZ), and PDB validation reports (PDF and $\mathrm{xml}$ ) for the $\mathrm{Mg}^{2+}$ complex and the BU72- $\mu \mathrm{OR}$ adduct; ligand distortions and $\mathrm{Z}$ scores (xlsx); molecular structures (cml); ideal structure (pdb) and restraints (mmCif) for the BU72-histidine adduct in isolation. An interactive visual comparison of the adduct and original model is available at:

\section{molstack.bioreproducibility.org/p/Y7FU}

Data availability: All data generated or analyzed during this study are included in the supplementary information files. Coordinates and structure factors for the adduct have been deposited in the PDB (7L0T).

\section{Bibliography}

1 Huang, W. et al. Structural insights into $\mu$-opioid receptor activation. Nature 524, 315-321 doi.org/f7m78z (2015).

2 Chan, H. C. S. et al. Enhancing the signaling of GPCRs via orthosteric ions. ACS Central Science 6, 274-282 doi.org/dk25 (2020).

3 Koehl, A. et al. Structure of the $\mu$-opioid receptor- $\mathrm{G}_{\mathrm{i}}$ protein complex. Nature 558, 547-552 doi.org/cqzg (2018).

$4 \quad$ Kruse, H., Sponer, J. \& Auffinger, P. Comment on "Evaluating unexpectedly short non-covalent distances in X-ray crystal structures of proteins with electronic structure analysis". Fournal of Chemical Information and Modeling 59, 3605-3608 doi.org/d8jz (2019).

$5 \quad$ Munro, T. A. Revised ( $\beta$-phenyl) stereochemistry of ultrapotent $\mu$ opioid BU72. bioRxiv doi.org/dq7s (2020).

6 Huang, W. et al. Author correction: Structural insights into $\mu$-opioid receptor activation. Nature 584, E16 doi.org/d5jij (2020).

7 Kuppuraj, G., Dudev, M. \& Lim, C. Factors governing metal-ligand distances and coordination geometries of metal complexes. Fournal of Physical Chemistry B 113, 2952-2960 doi.org/dqgnpj (2009).

8 Dudev, M., Wang, J., Dudev, T. \& Lim, C. Factors governing the metal coordination number in metal complexes from Cambridge Structural Database analyses. Fournal of Physical Chemistry B 110, 1889-1895 doi.org/dbw84h (2006).

9 Masson, J.-F., Gauda, E., Mizaikoff, B. \& Kranz, G. The interference of HEPES buffer during amperometric detection of ATP in clinical applications. Analytical and Bioanalytical Chemistry 390, 2067-2071 doi.org/ $\underline{\text { fhkf72 }}$ (2008). 
10 Ueda, J.-i., Ozawa, T., Miyazaki, M. \& Fujiwara, Y. SOD-like activity of complexes of nickel(II) ion with some biologically important peptides and their novel reactions with hydrogen peroxide. Inorganica Chimica Acta 214, 29-32 doi.org/d8dbw9 (1993).

11 Chaudhuri, B. N. et al. Crystal structure of imidazole glycerol phosphate synthase: a tunnel through a $(\beta / \alpha)_{8}$ barrel joins two active sites. Structure 9, 987-997 doi.org/ cjmk4z (2001).

12 Aurich, H. G. Nitroxides. Patai's Chemistry of Functional Groups, 313-370 doi.org/cp3635 (1989).

13 Ihara, H. et al. 2-Oxo-histidine-containing dipeptides are functional oxidation products. Fournal of Biological Chemistry 294, 1279-1289 doi.org/d7n6 (2019).

14 Zhou, Q. et al. Common activation mechanism of class A GPCRs. eLife 8, e50279 doi.org/fpcw (2019).

15 Che, T. et al. Structure of the nanobody-stabilized active state of the kappa opioid receptor. Cell 172, 55-67 doi.org/chz8 (2018). 